\title{
Untangling the relationship between fat distribution, nutritional status and Parkinson's disease severity
}

\author{
Maria S. Pisciotta ${ }^{1}$ - Domenico Fusco ${ }^{2}$ - Giulia Grande ${ }^{3}$. Vincenzo Brandi ${ }^{2} \cdot$ Maria R. Lo Monaco $^{2}$ - Alice Laudisio ${ }^{4}$. \\ Graziano Onder ${ }^{2} \cdot$ Anna R. Bentivoglio ${ }^{5,6}$. Diego Ricciardi ${ }^{2} \cdot$ Roberto Bernabei $^{2} \cdot$ Giuseppe Zuccalà $^{2}$. \\ Davide L. Vetrano ${ }^{2,3}$
}

Received: 17 November 2018 / Accepted: 28 February 2019 / Published online: 15 March 2019

(c) The Author(s) 2019

\begin{abstract}
Background Parkinson's disease (PD) is responsible for significant changes in body composition.

Aims We aimed to test the association between PD severity and fat distribution patterns, and to investigate the potential modifier effect of nutritional status in this association.

Methods We enrolled 195 PD subjects consecutively admitted to a university geriatric day hospital. All participants underwent comprehensive clinical evaluation, including assessment of total and regional body composition (dual-energy X-ray absorptiometry, DXA), body mass index, nutritional status (Mini-Nutritional Assessment, MNA), motor disease severity (UPDRS III), comorbidities, and pharmacotherapy.

Results The fully adjusted linear regression model showed a negative association between UPDRS III and total body fat in $\mathrm{kg}$ and percentage (respectively, $B-0.79 ; 95 \% \mathrm{CI}-1.54$ to -0.05 and $B-0.55 ; 95 \% \mathrm{CI}-1.04$ to -0.05 ), percentage android fat $(B-1.07 ; 95 \% \mathrm{CI}-1.75$ to -0.39$)$, trunk-leg fat ratio $(B-0.02 ; 95 \% \mathrm{CI}-0.04$ to -0.01$)$, trunk-limb fat ratio $(B-0.01 ; 95 \% \mathrm{CI}-0.06$ to -0.01$)$ and android-gynoid fat ratio $(B-0.01 ; 95 \% \mathrm{CI}-0.03$ to -0.01$)$. After stratification by MNA score, all the parameters of android-like fat distribution resulted negatively associated $(p<0.001$ for all) with UPDRS III, but only among subjects with a MNA $<23.5$ (risk of malnutrition or malnutrition).

Conclusion We found a negative association between severity of motor impairment and total fat mass in PD, more specific with respect to an android pattern of fat distribution. This association seems to be driven by nutritional status, and is significant only among patients at risk of malnutrition or with overt malnutrition.
\end{abstract}

Keywords Parkinson's disease $\cdot$ Body composition $\cdot$ DXA $\cdot$ Nutritional status $\cdot$ Fat

Maria S. Pisciotta and Domenico Fusco equally contributed to the study.

\section{Davide L. Vetrano}

davide.vetrano@ki.se

1 Department of Geriatrics, Fondazione Poliambulanza, Brescia, Italy

2 Department of Geriatrics, Centro di Medicina dell'Invecchiamento, Università Cattolica del Sacro Cuore di Roma and IRCCS Fondazione Policlinico "A. Gemelli", Rome, Italy

3 Department of Neurobiology, Care Sciences and Society, Aging Research Center, Karolinska Institutet and Stockholm

\section{Introduction}

Aging and neurodegenerative diseases are both associated with qualitative and quantitative changes in body composition (i.e., muscle, bone and fat mass) $[1,2]$, and specific

University, Tomtebodavägen 18A, Solna, Stockholm 17165, Sweden

4 Department of Geriatrics, Campus Bio-Medico University, Rome, Italy

5 Institute of Neurology, Università Cattolica del Sacro Cuore di Roma and IRCCS Fondazione Policlinico "A. Gemelli”, Rome, Italy

6 "Don Gnocchi" Foundation, Milan, Italy 
patterns of fat content and distribution have been described across neurodegenerative conditions [2]. People with Parkinson's disease (PD), for example, have been reported to have higher prevalence of overweight and central obesity (i.e., visceral fat) in the early disease stages as compared with healthy controls [3]. Conversely, weight loss as well as low body mass index (BMI) dominate the latest stages of the disease in PD [4, 5], and have been associated with nigrostriatal depletion [6], impaired motor function [7], poor quality of life [8], and cognitive impairment [9].

Loss of body fat mass has been advocated as the main responsible factor for weight loss in PD [4], but little is known about the potential role of nutritional status as a potentially amendable mediator of such phenomenon. PD is indeed characterized by an altered balance between energy intake and expenditures, and an increased resting energy expenditure has been described, possibly related to rigidity and levodopa-induced dyskinesias [10]. Malnutrition is common during the progression of PD, affecting up to $24 \%$ of patients. Using screening tools (such as Mini-Nutritional Assessment, MNA) an even higher proportion of PD patients are identified as at risk of developing malnutrition [11]. An inadequate nutritional status may affect the relation between motor disturbances and weight loss in PD.

Furthermore, anorexia, gastrointestinal symptoms (e.g., nausea, constipation or delayed gastric emptying), depression, cognitive decline, loss of independence in activities of daily living (such as preparing meals) due to worsening motor performance, might all concur to reduced energy intake [12].

The aims of the present study were (1) to investigate the association between PD severity and several parameters of adiposity, paying special attention to the topology of fat distribution (android vs gynoid); and (2) to address the potential modifier effect of nutritional status in the association between PD severity and fat distribution.

\section{Methods}

\section{Study participants}

In this cross-sectional study, we analyzed 195 PD subjects consecutively admitted to the geriatric day hospital of the Catholic University of Rome, Italy, between January 1st, 2012 and December 31st, 2015. From an original sample of 213 subjects, fourteen have been excluded because of missing data on body composition and four because of missing data on nutritional status. PD was diagnosed in keeping with the United Kingdom Parkinson's Disease Society Brain Bank criteria. Trained physicians evaluated all the participants. Data on demographics, functional status, diseases and drug treatment were properly collected. Fasting blood samples were obtained. All participants provided written informed consent, and the study protocol was previously approved from the Catholic University bioethics committee.

\section{Body composition evaluation}

Body weight $(\mathrm{Kg})$ and height $(\mathrm{m})$ were measured in standard conditions in all participants, and body mass index (BMI) was properly computed and expressed in $\mathrm{Kg} / \mathrm{m}^{2}$. All participants underwent body composition evaluation through dualenergy X-ray absorptiometry (DXA) on a daily automatic calibrated Hologic (Waltham, MA) system. Several parameters of total and regional adiposity were obtained through the automated embedded software algorithms [13]. We analyzed total fat (kg and \% of body weight), android fat (kg and $\%$ of total body fat), and gynoid fat ( $\mathrm{kg}$ and \% of total body fat). The following parameters have been also considered: trunk-leg fat ratio, trunk-limb fat ratio and android-gynoid fat ratio as automatically provided by the system. Higher values of such ratios express a higher android-like fat distribution. Of notice, fat measured from a DXA whole-body scan is highly correlated and linearly related to visceral fat measurements carried out through computed tomography [14].

\section{Nutritional status evaluation}

Participants' nutritional status was evaluated through the Mini-Nutritional Assessment (MNA) [15]. This tool includes 18 items evaluating anthropometric, functional, clinical and dietary parameters, composing a total score as high as 30 . According to MNA, scoring of $<23.5$ suggests a malnutrition risk, and a score of $<17$ overt malnutrition. MNA is a validated screening tool suited for the evaluation of the nutritional status in older adults. It has been shown to provide reliable evaluations across different populations and care settings and to be useful in predicting several outcomes [16]. Finally, MNA has been indicated as a reliable tool for the evaluation of malnutrition and risk of malnutrition in people with PD [17-19].

\section{Covariates}

Data on age, gender and education were collected through a standard questionnaire. Time from diagnosis of PD was expressed in years. The severity of PD was assessed using the Unified Parkinson's Disease Rating Scale (UPDRS) part III that evaluates the cardinal symptoms of the disease, thus staging the level of motor impairment. All the evaluations were carried out during the participant's "on" phase, within $2 \mathrm{~h}$ from the last antiparkinsonian drug administration. The L-dopa equivalent daily dose (LEDD) was finally obtained and indexed by body weight, transforming the 
antiparkinsonian drug daily doses in L-dopa equivalents through a previously described algorithm [20]. Cognition was assessed using the Mini-Mental State Examination (MMSE), scoring 0-30, with higher scores indicating better performance. Functional ability was estimated using the Katz's activities of daily living (ADLs), scoring 0-6, with lower scores indicating higher dependency. Depressive symptoms were assessed using the validated Italian version of the 15-item Geriatric Depression Scale (GDS). Fasting blood samples were obtained from all participants. In the present study, erythrocyte sedimentation rate (ESR; $\mathrm{mm} / \mathrm{s}$ ) was analyzed and used as an inflammatory parameter. Drugs were coded according to the Anatomical Therapeutic and Chemical codes. Diagnoses were coded according to the International Classification of Diseases, ninth edition, Clinical Modification codes.

\section{Statistical analysis}

Variables are presented as mean values \pm standard deviation (SD) or median and Inter Quartile Range (IQR) and absolute numbers and percentages (\%), and properly compared between participants with an UPDRS III below and above the median value (24, IQR 18-31). Analysis of variance (ANOVA) and Chi-square test were used to compare continuous and categorical variables normally distributed, respectively, and non-parametric tests were used for nonnormally distributed variables. The crude correlation $\left(R^{2}\right.$ coefficient of determination) between all the adiposity parameters and UPDRS III was reported in scatter-plot figures according to a MNA cut-off value of 23.5. The association ( $B$ and $95 \%$ confidence interval $[95 \% \mathrm{CI}]$ ) between all the adiposity parameters and UPDRS III (every five points) was tested through three different linear regression models adjusted for potential confounders. The additive statistical interaction between MNA and UPDRS III was tested and analyses subsequently stratified according to MNA values above and below the score of 23.5. A $p$ value of $<0.05$ was considered significant. Analyses were carried out through SPSS for Windows 18.0 (SPSS Inc., Chicago, IL).

\section{Results}

Among the study participants the mean age was $73.6 \pm 7.2$ years, $71(36 \%)$ were females and $56(29 \%)$ presented with a MNA score of $<23.5$. As shown in Table 1, compared with subjects with a UPDRS above the median, those with a UPDRS below the median were more likely male, more educated and presented with better cognitive function, mood, functional status, and nutritional status. Also, they presented with lower ESR values and comorbidities. As shown in Table 2, subjects with an UPDRS III below the median presented with higher trunk--leg and trunk-limb ratios. As shown in Table 3, according to the fully adjusted linear regression model, UPDRS III was negatively associated with total body fat in $\mathrm{kg}$ and as \% (respectively, $B-0.79 ; 95 \% \mathrm{CI}$ -1.54 to -0.05 and $B-0.55 ; 95 \% \mathrm{CI}-1.04$ to -0.05 ), android fat as $\%(B-1.07 ; 95 \% \mathrm{CI}-1.75$ to -0.39$)$, trunk-leg fat ratio $(B-0.02 ; 95 \% \mathrm{CI}-0.04$ to -0.01$)$, trunk-limb fat ratio $(B-0.01 ; 95 \% \mathrm{CI}-0.06$ to -0.01$)$, and android-gynoid fat ratio $(B-0.01 ; 95 \% \mathrm{CI}-0.03$ to -0.01$)$. After entering the MNA score in the adjusted model, only android fat as \% and trunk-leg fat ratio were still negatively associated with UPDRS III, but the association was weaker. Figures 1 and 2 show the correlation between all the adiposity parameters and UPDRS III stratified by the MNA. According to such analyses, all the parameters indicating an android-like fat distribution resulted negatively associated ( $p<0.001$ for all) with UPDRS III, but only among subjects with a MNA $<23.5$. The interaction analysis showed a statistical additive interaction between the UPDRS
Table 1 Main characteristics of participants according to the median UPDRS III score (below or above the median value)

\begin{tabular}{lllr}
\hline & UPDRS III $<24$ & UPDRS III $\geq 24$ & \multicolumn{1}{l}{$p$} \\
& $N=97(50 \%)$ & $N=98(50 \%)$ & \\
\hline Age, mean \pm SD & $73.1 \pm 6.7$ & $74.2 \pm 7.7$ & 0.261 \\
Sex (female), $n(\%)$ & $26(27)$ & $45(46)$ & 0.006 \\
Education (years), mean \pm SD & $11.9 \pm 4.7$ & $9.6 \pm 5.1$ & 0.001 \\
Mini-Mental State Examination, mean \pm SD & $27.2 \pm 2.2$ & $25.6 \pm 3.9$ & $<0.001$ \\
Geriatric Depression Scale, mean \pm SD & $4.6 \pm 3.0$ & $6.0 \pm 3.7$ & 0.004 \\
Activities of daily living, mean \pm SD & $5.1 \pm 1.0$ & $4.0 \pm 1.6$ & $<0.001$ \\
Mini-Nutritional Assessment, mean \pm SD & $25.4 \pm 3.0$ & $23.4 \pm 3.6$ & $<0.001$ \\
Erythrocyte sedimentation rate (mm/s), median (IQR) & $9(7-16)$ & $13(7-25)$ & 0.030 \\
Years from Parkinson's diagnosis, median $(\mathrm{IQR})$ & $2.9(0.8-6.8)$ & $4(1.9-7.8)$ & 0.400 \\
Levodopa equivalent daily dose $(\mathrm{mg} / \mathrm{kg})$, mean $\pm \mathrm{SD}$ & $8.0 \pm 5.7$ & $8.2 \pm 5.1$ & 0.741 \\
Number of chronic comorbidities, mean $\pm \mathrm{SD}$ & $3.0 \pm 1.8$ & $3.7 \pm 2.0$ & 0.007 \\
Number of drugs, mean \pm SD & $5.8 \pm 2.8$ & $6.2 \pm 2.9$ & 0.353 \\
\hline
\end{tabular}


Table 2 Association $(B$ and $95 \%$ confidence intervals) between the UPDRS III (every five-point increase) and adiposity parameters

\begin{tabular}{lccc}
\hline & UPDRS III $<24$ & UPDRS III $\geq 24$ & $P$ \\
& $N=97(50 \%)$ & $N=98(50 \%)$ & \\
\hline Body mass index $\left(\mathrm{kg} / \mathrm{m}^{2}\right)$, mean $\pm \mathrm{SD}$ & $27.4 \pm 4.0$ & $26.9 \pm 5.4$ & 0.503 \\
Total fat $(\mathrm{kg})$, mean $\pm \mathrm{SD}$ & $23.6 \pm 8.2$ & $22.4 \pm 8.5$ & 0.318 \\
Total fat $(\%)$, mean $\pm \mathrm{SD}$ & $30.8 \pm 6.3$ & $32.0 \pm 7.4$ & 0.260 \\
Android fat $(\mathrm{kg})$, mean $\pm \mathrm{SD}$ & $1.9 \pm 0.8$ & $1.8 \pm 0.9$ & 0.222 \\
Android fat $(\%)$, mean $\pm \mathrm{SD}$ & $33.4 \pm 7.0$ & $32.2 \pm 8.4$ & 0.297 \\
Gynoid fat $(\mathrm{kg})$, mean $\pm \mathrm{SD}$ & $3.4 \pm 1.0$ & $3.4 \pm 1.3$ & 0.962 \\
Gynoid fat $(\%)$, mean $\pm \mathrm{SD}$ & $31.8 \pm 6.1$ & $33.5 \pm 7.5$ & 0.081 \\
Trunk-leg ratio, mean $\pm \mathrm{SD}$ & $1.0 \pm 0.1$ & $0.9 \pm 0.2$ & 0.001 \\
Trunk-limb ratio, mean $\pm \mathrm{SD}$ & $1.3 \pm 0.3$ & $1.2 \pm 0.3$ & 0.016 \\
Android-gynoid fat ratio, mean $\pm \mathrm{SD}$ & $0.6 \pm 0.2$ & $0.5 \pm 0.2$ & 0.503 \\
\hline
\end{tabular}

${ }^{\text {a }}$ Percentage of the total region mass

Table 3 Association ( $B$ and 95\% confidence intervals) between the UPDRS III (every five-point increase) and adiposity parameters

\begin{tabular}{|c|c|c|c|c|c|c|c|c|c|}
\hline & \multicolumn{3}{|c|}{ Age, sex and education adj. } & \multicolumn{3}{|c|}{ Fully adj. $^{\mathrm{a}}$} & \multicolumn{3}{|c|}{ Fully adj + MNA } \\
\hline & \multirow[t]{2}{*}{$\mathrm{B}$} & \multicolumn{2}{|l|}{$95 \% \mathrm{CI}$} & \multirow[t]{2}{*}{$\mathrm{B}$} & \multicolumn{2}{|l|}{$95 \% \mathrm{CI}$} & \multirow[t]{2}{*}{$\mathrm{B}$} & \multicolumn{2}{|l|}{$95 \% \mathrm{CI}$} \\
\hline & & Lower limit & Upper limit & & Lower limit & Upper limit & & Lower limit & Upper limit \\
\hline Body mass index & -0.21 & -0.56 & 0.15 & -0.22 & -0.65 & 0.21 & -0.01 & -0.43 & 0.42 \\
\hline Total fat $(\mathrm{kg})$ & -0.70 & -1.31 & -0.09 & -0.79 & -1.54 & -0.05 & -0.51 & -1.26 & 0.24 \\
\hline Total fat $(\%)$ & -0.46 & -0.87 & -0.05 & -0.55 & -1.04 & -0.05 & -0.35 & -0.84 & 0.15 \\
\hline Android fat (kg) & -0.73 & -0.14 & -0.10 & -0.08 & -0.15 & 0.01 & -0.04 & -0.12 & 0.03 \\
\hline Android fat $\left(\%^{\mathrm{b}}\right)$ & -1.02 & -1.58 & -0.47 & -1.07 & -1.75 & -0.39 & -0.74 & -1.41 & -0.07 \\
\hline Gynoid fat (kg) & -0.06 & -0.14 & 0.02 & -0.09 & -0.19 & 0.01 & -0.05 & -0.15 & 0.05 \\
\hline Gynoid fat $\left(\%^{\mathrm{b}}\right)$ & -0.22 & -0.59 & 0.16 & -0.35 & -0.80 & 0.10 & -0.23 & -0.69 & 0.23 \\
\hline Trunk-leg fat & -0.02 & -0.04 & -0.01 & -0.02 & -0.04 & -0.01 & -0.02 & -0.03 & -0.01 \\
\hline Trunk-limb fat & -0.03 & -0.06 & -0.01 & -0.03 & -0.06 & -0.01 & -0.02 & -0.05 & 0.01 \\
\hline Android-gynoid fat & -0.02 & -0.03 & -0.01 & -0.01 & -0.03 & -0.01 & -0.01 & -0.02 & 0.01 \\
\hline
\end{tabular}

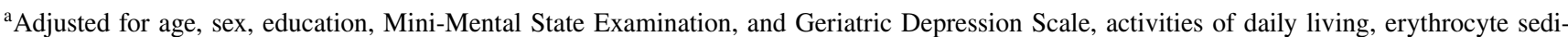
mentation rate, and number of comorbidities

${ }^{b}$ Percentage of total fat
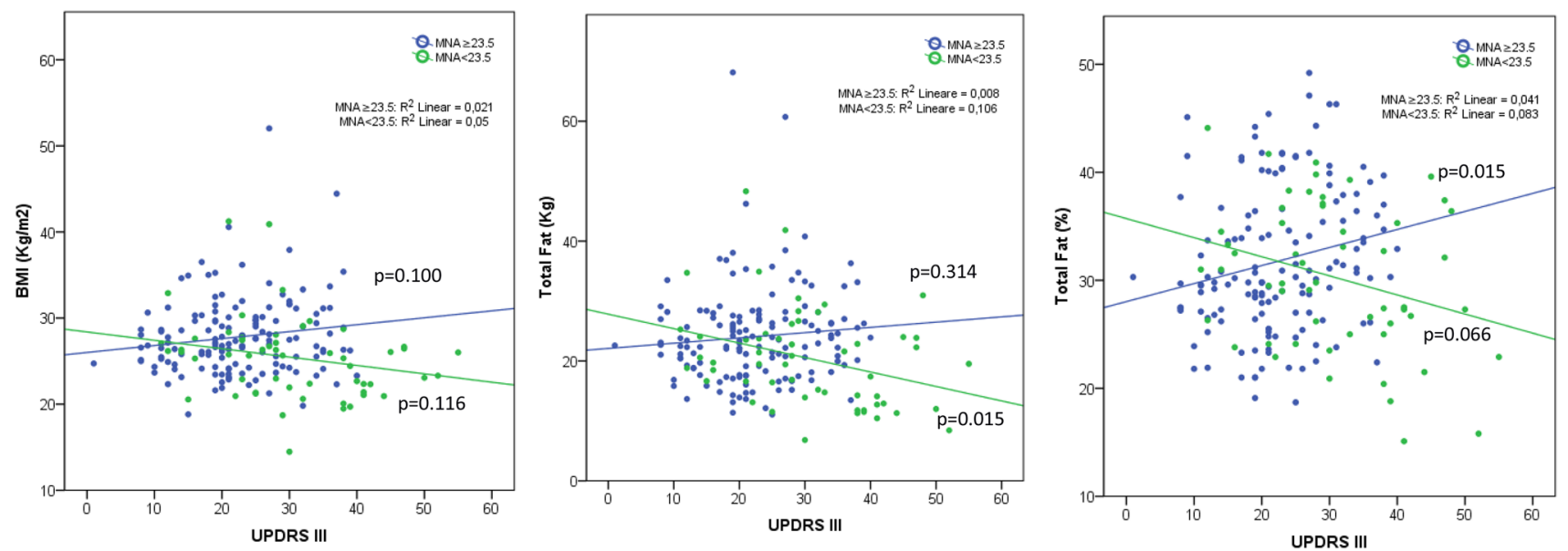

Fig. 1 Correlation between total body fat measures and UPDRS III by Mini-Nutritional Assessment score 

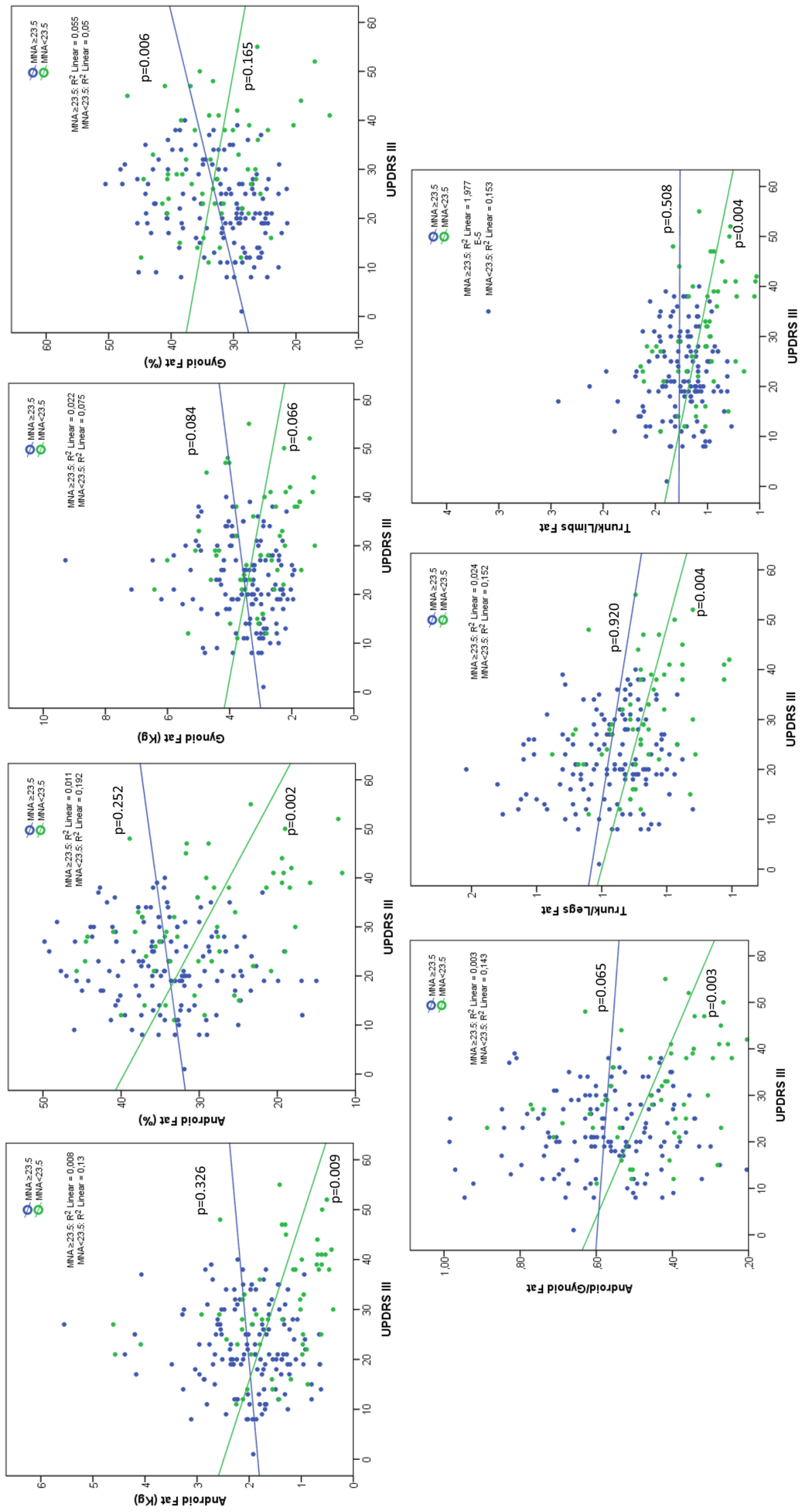

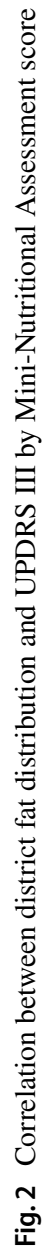


III and the MNA score for most of the adiposity parameters (see Table 4). In the stratified analyses, all the adiposity parameters resulted negatively associated with UPDRS III only among participants with a MNA score $<23.5$. Conversely, gynoid fat as $\%$ was positively associated with UPDRS III among subjects with a MNA $\geq 23.5$.

\section{Discussion}

In this sample of older adults with PD we observed a negative association between severity of motor impairment and fat mass, selectively with android-like distributed fat. The association remained significant after adjusting for several confounders, but not after adjusting for nutritional status. After stratification, the association between motor severity of PD and measures of adiposity was confirmed only among people at risk of malnutrition, or with overt malnutrition.

To the best of our knowledge, this is the largest sample of people with PD which body composition has been assessed through DXA scan. Weight loss is common in neurodegenerative diseases, and can be due to loss of energy balance. This may be related either to primary neuronal dysfunction and neurodegeneration (such as olfactory and taste loss, impairment of hypothalamic regulation of appetite and thermoregulation, cognitive decline, depression) or to secondary factors, such as disability and drugs' side effects [17, 21]. Weight loss in PD may present soon after the diagnosis; it has been related to loss of body fat [22], even if concomitant reduced lean mass cannot be excluded [23]. However, data on fat distribution through the course of disease are discordant. In the early stage of PD, an increase in body weight (mainly as visceral fat) has been reported [3]. Indeed, Bernhardt et al. recently demonstrated a higher visceral to sub-cutaneous fat ratio in parkinsonian subjects when compared to healthy controls, using MRI [24].

Generalized sympathetic denervation is common in PD [25-27], even in the early phase of disease. Autonomic dysfunction could be responsible for greater fat deposition since sympathetic denervation leads to chronotropic insufficiency and reduced thermogenesis, thus reducing energy expenditure [28]. Therefore, after an initial increase in body weight, a progressive weight loss occurs [4], more pronounced in the advanced stages of disease [19]. In our sample, worse motor performances (as assessed by UPDRS) were associated with reduced fat mass. This association was stronger with android fat. This is in line with previous observations [7, 11].

The main result of our study is that nutritional status drives the association between total and regional adiposity and disease severity in Parkinson's disease patients. Recent studies have suggested a potential neuroprotective role of adipocytokines. Reduced levels of leptin, a cytokine released by adipocytes, were observed by some authors in PD patients experiencing weight loss. Reduced levels of leptin reported in PD people with weight loss might explain the relationship between worse motor function and low fat mass [29, 30]. Beyond its contribution to body weight, the qualitative distribution of fat mass has several implications worthy to be mentioned. Android fat distribution has been related to increased cardiovascular disease risk, mediated by hyperlipidemia and hyperglycemia [31]. Indeed, insulin resistance has been associated with a slight increase in risk of PD, through a suppressive action on dopaminergic neurons in substantia nigra [32]. The reduced CV risk described
Table 4 Association ( $B$ and 95\% confidence intervals) between UPDRS III (every fivepoint increase) and adiposity parameters stratified by MNA

\begin{tabular}{|c|c|c|c|c|c|c|c|}
\hline & \multicolumn{3}{|c|}{$\begin{array}{l}\text { MNA } \leq 23.5 \\
N=56(29 \%)\end{array}$} & \multicolumn{3}{|c|}{$\begin{array}{l}\text { MNA > } 23.5 \\
N=139(71 \%)\end{array}$} & \multirow[t]{3}{*}{ Interaction $^{\mathrm{a}}$} \\
\hline & \multirow[t]{2}{*}{ B } & \multicolumn{2}{|l|}{$95 \% \mathrm{CI}$} & \multirow[t]{2}{*}{ B } & \multicolumn{2}{|l|}{$95 \% \mathrm{CI}$} & \\
\hline & & Lower limit & Upper limit & & Lower limit & Upper limit & \\
\hline Body mass index & -0.87 & -1.61 & -0.12 & 0.34 & -0.19 & 0.87 & 0.009 \\
\hline Total fat (kg) & -2.11 & -3.33 & -0.89 & 0.33 & -0.62 & 1.28 & 0.012 \\
\hline Total fat (\%) & -1.70 & -2.65 & -0.76 & 0.37 & -0.19 & 0.92 & $<0.001$ \\
\hline Android fat (kg) & -0.21 & -0.35 & -0.07 & 0.04 & -0.05 & 0.14 & 0.010 \\
\hline Android fat $\left(\%^{\ddagger}\right)$ & -2.51 & -3.77 & -1.25 & 0.10 & -0.66 & 0.86 & 0.001 \\
\hline Gynoid fat (kg) & -0.29 & -0.47 & -0.10 & 0.09 & -0.03 & 0.02 & 0.002 \\
\hline Gynoid fat $\left(\%^{\mathrm{b}}\right)$ & -1.65 & -2.57 & -0.72 & 0.58 & 0.10 & 1.07 & $<0.001$ \\
\hline Trunk-leg fat & -0.03 & -0.05 & -0.01 & -0.02 & -0.04 & 0.01 & 0.941 \\
\hline Trunk-limb fat & -0.06 & -0.10 & -0.02 & -0.01 & -0.04 & 0.03 & 0.372 \\
\hline Android-gynoid fat & -0.02 & -0.04 & -0.01 & -0.01 & -0.02 & 0.01 & 0.297 \\
\hline
\end{tabular}

Model adjusted for age, sex, education, Mini-Mental State Examination, Geriatric Depression Scale, activities of daily living, erythrocyte sedimentation rate, and number of comorbidities

${ }^{a}$ Additive interaction ( $p$ value) between the Mini-Nutritional Assessment and UPDRS III

${ }^{\mathrm{b}}$ Percentage of total fat 
in people with $\mathrm{PD}$, and associated with both disease severity and duration, may be partially mediated by the reduction in android adiposity we describe in the present paper, at least for subjects at risk of malnutrition.

Like weight loss, malnutrition is common in PD [11] and many possible causes can contribute to its occurrence: hyposmia, reduced appetite, altered reward mechanism due to degeneration in meso-corticolimbic network, reduced levels of orexin, could all account for undernutrition, but none of them has been consistently related to weight loss [33]. Increased energy expenditure has been observed in PD patients with worsening motor performance [34], and its main determinants are considered dyskinesias and rigidity. Along with gastrointestinal symptoms (such as sialorrhea, dysphagia and constipation), leading to reduced energy intake and malnutrition [12], disability, cognitive impairment, and depression are also significant risk factors for malnutrition in the elderly population and should be taken into consideration [35].

In our study, after adjusting for possible confounders, the association of UPDRS and fat mass remained statistically significant only for the subgroup of patients with lower MNA scores. In this group, higher fat mass (particularly android fat) could represent an index of better nutritional status. In other words, a good nutritional status might protect PD patients from weight loss associated with disease severity. Maintaining a good nutritional status might potentially slow both weight loss and motor impairment. Screening tools, such as MNA, may allow early detection of subjects with PD at risk for malnutrition and proper personalized nutritional intervention [16, 17]. Individualized dietary counseling should be offered to patients, taking into consideration the specific clinical context, disease duration, level of motor impairment and comorbidities.

To note, a cross-talk between fat and muscle has been demonstrated by several studies. A number of signaling proteins, produced by muscle and fat cells take part to this talk and are responsible for the changes in body composition observed with age [36]. Interestingly, in older people, along with body weight changes, a common consequence of malnutrition is represented by sarcopenia, the progressive decline in muscle mass and muscle function. In a recent study carried out on the same study population of the present report, we showed that in PD patients, sarcopenia is associated with more than twofold higher odds of poor motor function, as measured through the UPDRS [37]. The potential synergism between muscle and fat changes and their impact on motor function in PD requires to be addressed in future longitudinal studies.

Some limitations of the present study need to be acknowledged. First, as previously stated, the cross-sectional nature does not allow firm conclusions either on causality or timing among the observed phenomena. Second, we analyzed a non-random sample of elderly people with $\mathrm{PD}$, admitted to a single care center; this may limit the generalizability of our results. Third, functional or biological parameters not collected in the present study might account for a residual confounding, potentially affecting our results.

In conclusion, our results suggest that nutritional status plays a relevant role in driving the relationship between body composition (particularly fat content and distribution) and motor function in people with PD. The detrimental effect of weight loss on motor function might be prevented by maintaining a proper nutritional status, avoiding malnutrition. In this regard, the early detection of malnutrition or risk of malnutrition in subjects with PD is warranted. Such findings deserve further investigation through longitudinal studies.

Author contributions DF, MSP, DLV, and GZ designed the study. DLV, MSP, VB, MRLM, DF, DR collected the data. DLV, GO, GG and $\mathrm{AL}$ analyzed the data. DF, MSP and DLV wrote the first draft. ARB, GG, RB and GZ gave their contribution to the manuscript according their specific expertise. All the authors shared and accepted the last manuscript draft.

Funding None.

\section{Compliance with ethical standards}

Conflict of interest The authors declare that they have no conflict of interest.

Ethical approval The study protocol was previously approved from the Catholic University bioethics committee and all procedures performed in studies involving human participants were in accordance with the 1964 Helsinki declaration and its later amendments or comparable ethical standards.

Informed consent All participants provided written informed consent.

Open Access This article is distributed under the terms of the Creative Commons Attribution 4.0 International License (http://creativeco mmons.org/licenses/by/4.0/), which permits unrestricted use, distribution, and reproduction in any medium, provided you give appropriate credit to the original author(s) and the source, provide a link to the Creative Commons license, and indicate if changes were made.

\section{References}

1. Reinders I, Visser M, Schaap L (2017) Body weight and body composition in old age and their relationship with frailty. Curr Opin Clin Nutr Metab Care 20:11-15

2. Diehl-Wiesenecker E, von Armin CA, Dupuis L et al (2015) Adipose tissue distribution in patients with Alzheimer's Disease: a whole body MRI case-control study. J Alzheimers Dis 48:825-832

3. Vikdahl M, Carlsson M, Linder J et al (2014) Weight gain and increased central obesity in the early phase of Parkinson's disease. Clin Nutr 33:1132-1139 
4. Chen H, Zhang SM, Hernán MA et al (2003) Weight loss in Parkinson's disease. Ann Neurol 53:676-679

5. Van der Marck MA, Dicke HC, Uc EY et al (2012) Body mass index in Parkinson's disease: a meta-analysis. Parkinsonism Relat Disord 18:263-267

6. Lee JJ, Oh JS, Ham JH et al (2016) Association of body mass index and the depletion of nigrostriatal dopamine in Parkinson's disease. Neurobiol Aging 38:197-204

7. Wills AM, Pérez A, Wang J et al (2016) Association between change in Body Mass Index, unified Parkinson's disease Rating Scale Scores, and survival among persons with Parkinson Disease: secondary analysis of longitudinal data from NINDS exploratory trials in parkinson disease long-term study 1. JAMA Neurol 73:321-328

8. Akbar U, He Y, Dai Y et al (2015) Weight loss and impact on quality of life in Parkinson's disease. PLoS One 10:e0124541

9. Kim HJ, Oh ES, Lee JH et al (2012) Relationship between changes of body mass index (BMI) and cognitive decline in Parkinson's disease (PD). Arch Gerontol Geriatr 55:70-72

10. Capecci M, Petrelli M, Emanuelli B et al (2013) Rest energy expenditure in Parkinson's disease: role of disease progression and dopaminergic therapy. Parkinsonism Relat Disord 19:238-241

11. Sheard JM, Ash S, Mellick GD et al (2013) Markers of disease severity are associated with malnutrition in Parkinson's disease. PLoS One 8:e57986

12. Barichella M, Cereda E, Madio C et al (2013) Nutritional risk and gastrointestinal dysautonomia symptoms in Parkinson's disease outpatients hospitalised on a scheduled basis. Br J Nutr 28:347-353

13. Toombs RJ, Ducher G, Shepherd JA et al (2012) The impact of recent technological advances on the trueness and precision of DXA to assess body composition. Obesity (Silver Spring) 20:30 39. https://doi.org/10.1038/oby.2011.211

14. Micklesfield LK, Goedecke JH, Punyanitya M et al (2012) Dualenergy X-ray performs as well as clinical computed tomography for the measurement of visceral fat. Obesity 20:1109-1114

15. Guigoz Y, Vellas B, Garry PJ (1996) Assessing the nutritional status of the elderly: the mini nutritional assessment as part of the geriatric evaluation. Nutr Rev 55:59-65

16. Cereda E, Pedrolli C, Klersy C et al (2016) Nutritional status in older persons according to healthcare setting: a systematic review and meta-analysis of prevalence data using MNA (囚). Clin Nutr https://doi.org/10.1016/j.clnu.2016.03.008

17. Laudisio A, Vetrano DL, Meloni E et al (2014) Dopaminergic agents and nutritional status in Parkinson's disease. Mov Disord 29:1543-1547. https://doi.org/10.1002/mds.25991

18. Fereshtehnejad SM, Ghazi L, Sadeghi M et al (2014) Prevalence of malnutrition in patients with Parkinson's disease: a comparative study with healthy controls using Mini Nutritional Assessment (MNA) questionnaire. J Parkinsons Dis 4:473-481. https://doi. org/10.3233/JPD-130323

19. Barichella M, Cereda E, Pezzoli G (2009) Major nutritional issues in the management of Parkinson's disease. Mov Disord 24:1881-1892

20. Tomlinson CL, Stowe R, Patel S et al (2010) Systematic review of levodopa dose equivalency reporting in Parkinson's disease. Mov Disord 25:2649-2653

21. Aziz NA, van der Marck MA, Pijl H et al (2008) Weight loss in neurodegenerative disorders. J Neurol 255:1872-1880. https://doi. org/10.1007/s00415-009-0062-8
22. Beyer PL, Palarino MY, Michalek D et al (1995) Weight change and body composition in patients with Parkinson's disease. J Am Diet Assoc 95:979-983

23. Barichella M, Pinelli G, Iorio L et al (2016) Sarcopenia and dynapenia in patients with Parkinsonism. J Am Med Dir Assoc 17:640-646

24. Bernhardt D, Müller HP, Ludolph AC et al (2016) Body fat distribution in Parkinson's disease: an MRI-based body fat quantification study. Parkinsonism Relat Disord. https://doi.org/10.1016/j. parkreldis.2016.09.016

25. Asahina M, Vichayanrat E, Low DA et al (2013) Autonomic dysfunction in parkinsonian disorders: assessment and pathophysiology. J Neurol Neurosurg Psychiatry 84:674-680. https://doi. org/10.1136/jnnp-2012-303135

26. Vetrano DL, Pisciotta MS, Brandi V et al (2016) Impact of disease duration and cardiovascular dysautonomia on hypertension in Parkinson's disease. J Clin Hypertens (Greenwich). https://doi. org/10.1111/jch.12938

27. Vetrano DL, Pisciotta MS, Lo Monaco MR et al (2015) Association of depressive symptoms with circadian blood pressure alterations in Parkinson's disease. J Neurol 262:2564-2571. https://doi. org/10.1007/s00415-015-7887-0

28. Mochizuki H, Taniguchi A, Nakazato Y et al (2016) Increased body mass index associated with autonomic dysfunction in Parkinson's disease. Parkinsonism Relat Disord 24:129-131

29. Evidente VG, Caviness JN, Adler CH et al (2001) Serum leptin concentrations and satiety in Parkinson's disease patients with and without weight loss. Mov Disord 16:924-927

30. Lorefält B, Toss G, Granérus AK (2009) Weight loss, body fat mass, and leptin in Parkinson's disease. Mov Disord 24:885-890

31. Matsuzawa Y (2008) The role of fat topology in the risk of disease. Int J Obes (Lond) 32:S83-S92. https://doi.org/10.1038/ ijo. 2008.243

32. Aviles-Olmos I, Limousin P, Lees A et al (2013) Parkinson's disease, insulin resistance and novel agents of neuroprotection. Brain 136:374-384. https://doi.org/10.1093/brain/aws009

33. Kim SR, Chung SJ, Yoo SH (2016) Factors contributing to malnutrition in patients with Parkinson's disease. Int J Nurs Pract 22:129-137. https://doi.org/10.1111/ijn.12377

34. Levi S, Cox M, Lugon M et al (1990) Increased energy expenditure in Parkinson's disease. BMJ 301:1256-1257

35. Moreira NC, Krausch-Hofmann S, Matthys C et al (2016) Risk factors for malnutrition in older adults: a systematic review of the literature based on longitudinal data. Adv Nutr 16:507-522

36. Indrakusuma I, Sell H, Eckel J (2015) Novel mediators of adipose tissue and muscle crosstalk. Curr Obes Rep 4:411-417. https:// doi.org/10.1007/s13679-015-0174-7

37. Vetrano DL, Pisciotta MS, Laudisio A et al (2018) Sarcopenia in parkinson disease: comparison of different criteria and association with disease severity. J Am Med Dir Assoc 19:523-527. https:// doi.org/10.1016/j.jamda.2017.12.005

Publisher's Note Springer Nature remains neutral with regard to jurisdictional claims in published maps and institutional affiliations. 